Supporting Information for:

\title{
Fourier-Transform Approach for Reconstructing Macromolecular Mass Defect Profiles
}

\author{
Andrew K. Swansiger, ${ }^{1}$ Michael T. Marty, ${ }^{2}$ and James S. Prell ${ }^{* 1,3}$ \\ Submitted to Journal of the American Society for Mass Spectrometry \\ 21 October 2021
}

Revised 30 November 2021

1 Department of Chemistry and Biochemistry, 1253 University of Oregon, Eugene, OR 974031253, USA

2 Department of Chemistry and Biochemistry, 1306 University of Arizona, Tucson, AZ 85721, USA

3 Materials Science Institute, 1252 University of Oregon, Eugene, OR 97403-1252, USA

*Correspondence should be addressed to jprell@uoregon.edu

Tel: (541) 346-2597

Fax: (541) 346-4643 


\section{Table of Contents:}

\begin{tabular}{|l|c|}
\hline $\begin{array}{l}\text { Mathematical proof of Fourier Transform elimination of global (envelope) phase } \\
\text { information allowing for extraction of local (peak) phase information }\end{array}$ & S-3 \\
\hline $\begin{array}{l}\text { Table S1: Table of UniDec parameters used for Bayesian deconvolution of Melittin- } \\
\text { embedded Nanodiscs }\end{array}$ & S-6 \\
\hline $\begin{array}{l}\text { Table S2: Table of UniDec parameters used for Bayesian deconvolution of mixed- } \\
\text { MSP1D1 Nanodiscs }\end{array}$ & $\mathbf{S - 7}$ \\
\hline $\begin{array}{l}\text { Table S3: Table of simulated results from two integration approximations for } \\
\text { statistical analysis of method accuracy }\end{array}$ & $\mathbf{S - 8}$ \\
\hline $\begin{array}{l}\text { Table S4: Table of relative mass defect errors of UniDec and iFAMS from expected } \\
\text { values based on empirical mass measurements for melittin and mixed belt data }\end{array}$ & $\mathbf{S - 9}$ \\
\hline $\begin{array}{l}\text { Figure S1: Simulation of the effects of different levels of mass spectral peak } \\
\text { superposition on mass defect resolution }\end{array}$ & $\mathbf{S - 1 1}$ \\
\hline $\begin{array}{l}\text { Figure S2: Comparison of MMD profile baseline correction done before and after } \\
\text { charge-state-specific profile summation }\end{array}$ & $\mathbf{S - 1 2}$ \\
\hline $\begin{array}{l}\text { Figure S3: ESI-MS spectrum of MSP1D1(-) demonstrating prevalence of both } \\
\text { monomeric and dimeric MSPs in solution }\end{array}$ & $\mathbf{S - 1 3}$ \\
\hline $\begin{array}{l}\text { Figure S4: ESI-MS spectrum of MSP1D1His demonstrating prevalence of both } \\
\text { monomeric and dimeric MSPS in solution }\end{array}$ & $\mathbf{S - 1 4}$ \\
\hline
\end{tabular}




\section{Mathematical Proof}

Throughout the following proof, we denote a function $f(y)$ that depends parametrically on quantities $a, b, \ldots$, as $f(y ; a, b, \ldots)$. We also denote the mass-to-charge ratio $m / z$ by $x$ for typographical clarity, and $k$ is defined as the frequency variable conjugate to $x$ in the frequency domain. $M_{L}$ denotes the mass of the repeated subunit/ligand in the ion population that gives rise to periodic signals in the frequency domain.

Let $\mathrm{T}_{\alpha}$ denote a translation operator that translates a function to the right by $\alpha$, e.g., $\mathrm{T}_{\alpha}\{f(y)\}=$ $f(y-\alpha)$. Let $c\left(x ; \frac{M_{L}}{Z}\right)$ denote a comb function of $x$ with peak spacing $\frac{M_{L}}{Z}$ (with a peak at $x=0$ ). Let $e\left(x ; x_{\max }(Z), Z\right)$ denote a slowly-varying envelope function of $x$ with maximum, $x_{\max }(Z)$. Finally, let $p(x ; Z)$ be a peak shape function, defined between $x=0$ and $x=\frac{M_{L}}{Z}$. (It is trivial to account later for additional mass due to a charge carrier, such as a proton, in defining $p(x ; Z)$; see below.)

The type of mass spectrum under consideration here is any such one that can be decomposed into the sum of charge-state-specific mass spectra $s(x ; Z)=\left[c\left(x ; \frac{M_{L}}{Z}\right) \times e\left(x ; x_{\max }(Z), Z\right)\right] *$ $p(x ; Z)$, with $Z \in\left\{Z_{\min }, Z_{\min }+1, \ldots, Z_{\max }\right\}$ the range of observed charge states. It is assumed that $e\left(x ; x_{\max }(Z), Z\right)$ and $x_{\max }(Z)$ may be different for each $Z$. Without loss of generality, then, $e\left(x ; x_{\max }(Z), Z\right)$ can be defined such that $x_{\max }(Z)$ coincides with a peak of $c\left(x ; \frac{M_{L}}{Z}\right)$, i.e., $x_{\max }(Z)$ is an integer multiple of $\frac{M_{L}}{Z}$. Emphatically, reconstructing the Macromolecular Mass Defect (MMD) profile for a particular charge state $Z$ is equivalent to recovering $p(x ; Z)$ from observation of $s(x ; Z)$. (Optionally, if we furthermore assume that the mass distribution represented by $p(x ; Z)$ is identical for all observed charge states, this amounts to recovering a single peak shape function $p(x)$ common to all $Z$ by summing over or otherwise averaging over the individual $p(x ; Z)$ after normalizing the $x$-axis for charge state.)

To determine $p(x ; Z)$ from a charge-state-specific mass spectrum, we first note that

$$
s(x ; Z)=\left[c\left(x ; \frac{M_{L}}{Z}\right) \times \mathrm{T}_{x_{\max }(Z)}\{e(x ; 0, Z)\}\right] * p(x ; Z)
$$

By the Fourier Convolution Theorem,

$$
S(k ; Z)=\left[C\left(k ; \frac{Z}{M_{L}}\right) * e^{-2 \pi i k x_{\max }(Z)} E(k ; 0, Z)\right] \times P(k ; Z)
$$

and we note that $k x_{\max }(Z)$ is an integer at each peak in the comb $C\left(k ; \frac{Z}{M_{L}}\right)$ by choice of $x_{\max }(Z)$. 
For sufficiently broad $e(x ; 0, Z), E(k ; 0, Z)$ is narrow and decays to zero within $\frac{1}{M_{L}}$ either side of $k=0$. In this case, we shall show that, by sampling this function at integer multiples of $\frac{Z}{M_{L}}$ (i.e., where the magnitude of $S(k ; Z)$ achieves local maxima), one can reconstruct $p(x ; Z)$. This can be difficult to do with the function in the form shown above, however, because $e^{-i k x_{\max }(Z)}$ generally contributes rapid phase oscillations to $S(k ; Z)$ that make it practically very difficult to determine phase at specific values of $k$ for realistic data.

To improve the situation, we first sample $S(k ; Z)$ by multiplying $S(k ; Z)$ by another copy of $C\left(k ; \frac{Z}{M_{L}}\right)$ :

$$
S(k ; Z) \times C\left(k ; \frac{Z}{M_{L}}\right)=C\left(k ; \frac{Z}{M_{L}}\right) \times\left[C\left(k ; \frac{Z}{M_{L}}\right) * e^{-i k x_{\max }(Z)} E(k ; 0, Z)\right] \times P(k ; Z)
$$

On the right hand side of this equation, multiplication by the comb has the effect of sampling $\left[C\left(k ; \frac{Z}{M_{L}}\right) * e^{-i k x_{\max }(Z)} E(k ; 0, Z)\right]$ at values of $k$ that are integer multiples of $\frac{Z}{M_{L}}$, where $\left[C\left(k ; \frac{Z}{M_{L}}\right) * e^{-i k x_{\max }(Z)} E(k ; 0, Z)\right]=E(0 ; 0, Z) \times e^{-i k x_{\max }(Z)}$.

Thus, at the sampled values of $k, S(k ; Z)=E(0 ; 0, Z) \times e^{-i k x_{\max }(Z)} \times P(k ; Z)$, or, equivalently,

$$
S(k ; Z) \times \frac{e^{i k x_{\max }(Z)}}{E(0 ; 0, Z)}=P(k ; Z)
$$

Note that this function has stationary (i.e., very slowly varying) phase due to the nearly even symmetry of $e(x ; 0, Z)$. That is, the phase of this function at the sampled values of $k$ depends only on $P(k ; Z)$, because we have eliminated the (generally, rapidly oscillating) contributions from the envelope function, $E\left(k ; x_{\max }(Z), Z\right)$. This is the key strategy that enables practical MMD profile reconstruction using the FT-based method, for the phase at peaks in the frequency domain is now relatively easy to see visually and measure accurately. In other words, sampling the Fourier Transform of the mass spectral signal, modulated by the scaled exponential on the left-hand side of this equation, is equivalent to sampling $P(k ; Z)$.

Now, $E(0 ; 0, Z)$ is equal to the integral over $e\left(x ; x_{\max }(Z)\right)$, which we denote $\bar{e}(Z)$, thus it is proportional to the total ion population with charge state $Z$, divided by $Z$. Because the spacing of the frequency-domain samples is $\frac{Z}{M_{L}}$, the inverse Fourier Transform of the sampled data set spans the interval $x \in\left[0, \frac{M_{L}}{Z}\right)$, the same as the interval on which $p(x ; Z)$ is defined.

Taking the inverse Fourier Transform of both sides, we have, on the interval $x \in\left[0, \frac{M_{L}}{Z}\right)$ :

$$
\mathrm{T}_{-x_{\max }(Z)}\{s(x ; Z)\} / \bar{e}(Z)=p(x ; Z)
$$

defined at a set of equally spaced points equal to the number of samples (i.e., harmonics used) in the frequency domain. 
Equivalently,

$$
\mathrm{T}_{-x_{\max }(Z)}\{s(x ; Z)\}=\bar{e}(Z) \times p(x ; Z)
$$

This is a reconstruction of $p(x ; Z)$ weighted by the total ion population for charge state $Z$ divided by $Z$, i.e., it is the MMD profile for charge state $Z$, the main target for reconstruction.

To combine $p(x ; Z)$ from all observed values of $Z$ into a single charge-state-averaged MMD profile $p(x)$, one can simply translate each $p(x ; Z)$ cyclically to the left by (mass of the charge carrier/1), normalize for charge by replacing $x$ by $Z x$, and interpolate and sum the resulting "zero-charge" MMD profiles over the set of all observed $Z$. Note that the axis transformation $x \mapsto Z x$ naturally accounts for the $1 / Z$ scaling of $e\left(x ; x_{\max }(Z), Z\right)$ such that each charge state's contribution to the summed MMD profile $(p(x))$ is simply proportional to its total ion population.

In summary, the FT-based MMD reconstruction presented here is based on the following idea: we take advantage of the Stationary Phase Approximation to confidently determine the phase contribution of $P(k ; Z)$, which is what is needed to reconstruct $p(x ; Z)$. 


\begin{tabular}{|c|c|c|c|c|c|}
\hline Data Processing & & & $\begin{array}{l}\text { Peak selection/ } \\
\text { extraction/plotting }\end{array}$ & & \\
\hline $\mathrm{m} / \mathrm{z}$ range & 1979.925 & 1000000 & Picking range & 50000 & \\
\hline Background subtraction & 0 & & Picking threshold & 0.1 & \\
\hline Bin every & 0 & & Peak normalization type & Max & \\
\hline Normalize data? & Yes & & Peak extraction method & Height & \\
\hline UniDec Params & & & Extraction window & 0 & \\
\hline Charge range & 10 & 18 & Extraction Threshold & 10 & \\
\hline Mass range & 100000 & 200000 & Extraction normalization type & Max & \\
\hline Sample mass every & 10 & & Add'tl Plotting Params & & \\
\hline Quick Controls & & & 2D color map type & nipy_spectral & \\
\hline $\begin{array}{l}\text { Smooth charge state } \\
\text { distribution? }\end{array}$ & Yes & & Peaks color map type & rainbow & \\
\hline Auto $\mathrm{m} / \mathrm{z}$ peak width? & Yes & & Spectra color map type & rainbow & \\
\hline $\begin{array}{l}\text { Level of Nearby-Point } \\
\text { Smoothing }\end{array}$ & Some & & Discrete plot? & No & \\
\hline Level of artifact suppression & None & & $\begin{array}{l}\text { Publication mode? } \\
\text { (Richardson-Lucy peak } \\
\text { sharpening) }\end{array}$ & No & \\
\hline Mass defect & 666.9 & & $\begin{array}{l}\text { Reconvolve w/peakshape or } \\
\text { raw }\end{array}$ & Raw & \\
\hline Add'tl Deconv Params & & & Marker threshold & 0.1 & \\
\hline Peak FWHM & 10 & & Species separation & 0.025 & \\
\hline Peak shape function & Gaussian & & Integration range & -1000 & 1000 \\
\hline Beta & 0 & & Limits on \# of spectra & 100 & 20 \\
\hline Charge smooth width & 1 & & Mass Defect Analysis & & \\
\hline Point smooth width & 1 & & Number of Defect Bins & 50 & \\
\hline Mass smooth width & 1 & & Subunit mass & 666.9 & \\
\hline Native charge offset range & -100 & 100 & $\mathrm{X}$-axis & mass(Da) & \\
\hline $\begin{array}{l}\text { Isotopes (isotopic resolution } \\
\text { only) }\end{array}$ & Off & & & & \\
\hline Manual mode & No & & & & \\
\hline Negative mode & No & & & & \\
\hline Charge scaling & No & & & & \\
\hline Mass list window & $\mathrm{N} / \mathrm{A}$ & & & & \\
\hline $\begin{array}{l}\mathrm{m} / \mathrm{z} \text { to mass transformation } \\
\text { type }\end{array}$ & Interpolate & & & & \\
\hline $\begin{array}{l}\text { Maximum number of } \\
\text { iterations }\end{array}$ & 100 & & & & \\
\hline Adduct mass & 1.007276 & & & & \\
\hline
\end{tabular}

Table S1 List of all UniDec parameters of used for spectral deconvolution of melittin-embedded Nanodisc data, in order as they appear in MetaUniDec Version 4.2.1. Richardson-Lucy peak sharpening reconstructions to recreate published profiles consisted of selecting publication mode, changing "Nearby Point Smoothing" to "None" and setting "Point Smooth Width" to zero. 


\begin{tabular}{|c|c|c|c|c|c|}
\hline Data Processing & & & $\begin{array}{l}\text { Peak selection/ } \\
\text { extraction/plotting }\end{array}$ & & \\
\hline $\mathrm{m} / \mathrm{z}$ range & Full spectrum & & Picking range & 500 & \\
\hline Background subtraction & 0 & & Picking threshold & 0.1 & \\
\hline Bin every & 0 & & Peak normalization type & Max & \\
\hline Normalize data? & Yes & & Peak extraction method & Height & \\
\hline UniDec Params & & & Extraction window & 0 & \\
\hline Charge range & $9,10,11$ & $15,17,18$ & Extraction Threshold & 10 & \\
\hline Mass range & 8000 & 300000 & Extraction normalization type & Max & \\
\hline Sample mass every & 10 & & Add'tl Plotting Params & & \\
\hline Quick Controls & & & 2D color map type & nipy_spectral & \\
\hline $\begin{array}{l}\text { Smooth charge state } \\
\text { distribution? }\end{array}$ & Yes & & Peaks color map type & rainbow & \\
\hline Auto $\mathrm{m} / \mathrm{z}$ peak width? & Yes & & Spectra color map type & rainbow & \\
\hline $\begin{array}{l}\text { Level of Nearby-Point } \\
\text { Smoothing }\end{array}$ & Other & & Discrete plot? & No & \\
\hline Level of artifact suppression & None & & $\begin{array}{l}\text { Publication mode? } \\
\text { (Richardson-Lucy peak } \\
\text { sharpening) }\end{array}$ & No & \\
\hline Mass defect & 677.9 & & $\begin{array}{l}\text { Reconvolve w/peakshape or } \\
\text { raw }\end{array}$ & Raw & \\
\hline Add'tl Deconv Params & & & Marker threshold & 0.1 & \\
\hline Peak FWHM & 0.85 & & Species separation & 0.025 & \\
\hline Peak shape function & Gaussian & & Integration range & -1000 & 1000 \\
\hline Beta & 0 & & Limits on \# of spectra & 100 & 20 \\
\hline Charge smooth width & 1 & & Mass Defect Analysis & & \\
\hline Point smooth width & 100 & & Number of Defect Bins & 50 & \\
\hline Mass smooth width & 1 & & Subunit mass & 677.9 & \\
\hline Native charge offset range & -100 & 100 & $\mathrm{X}$-axis & $\operatorname{mass}(\mathrm{Da})$ & \\
\hline $\begin{array}{l}\text { Isotopes (isotopic resolution } \\
\text { only) }\end{array}$ & Off & & & & \\
\hline Manual mode & No & & & & \\
\hline Negative mode & No & & & & \\
\hline Charge scaling & No & & & & \\
\hline Mass list window & $\mathrm{N} / \mathrm{A}$ & & & & \\
\hline $\begin{array}{l}\mathrm{m} / \mathrm{z} \text { to mass transformation } \\
\text { type }\end{array}$ & Smart & & & & \\
\hline $\begin{array}{l}\text { Maximum number of } \\
\text { iterations }\end{array}$ & 100 & & & & \\
\hline Adduct mass & 1.007276 & & & & \\
\hline
\end{tabular}

Table S2 List of all UniDec parameters of used for spectral deconvolution of melittin-embedded Nanodisc data, in order as they appear in UniDec Version 4.2.1. 


\begin{tabular}{|c|c|c|c|c|c|}
\hline $\begin{array}{l}\text { Single-Peak } \\
\text { Assumption }\end{array}$ & $\begin{array}{c}\text { Relative } \\
\text { Abundance }\end{array}$ & $1 / 1$ & $0.5 / 1$ & $0.25 / 1$ & $0.125 / 1$ \\
\hline \multicolumn{6}{|l|}{$\begin{array}{c}\text { Peak } \\
\text { Separation }\end{array}$} \\
\hline $0 \sigma$ & & $0.00 / 0.00$ & $0.00 / 0.00$ & $0.00 / 0.00$ & $0.00 / 0.00$ \\
\hline $1 \sigma$ & & $-50.00 / 50.00$ & $-66.67 / 33.33$ & $-80.00 / 20.00$ & $-88.89 / 11.11$ \\
\hline $2 \sigma$ & & $-100.00 / 100.00$ & $-133.33 / 66.67$ & $-160.00 / 40.00$ & $-177.78 / 22.22$ \\
\hline $3 \sigma$ & & $6.06 /-5.66$ & $20.89 / 3.89$ & $48.25 / 11.63$ & $-266.67 / 33.33$ \\
\hline $4 \sigma$ & & $1.81 /-1.59$ & $4.87 / 0.27$ & $9.29 / 1.25$ & $15.65 / 1.68$ \\
\hline $5 \sigma$ & & $0.44 /-0.36$ & $1.17 / 0.06$ & $2.13 / 0.22$ & $3.68 / 0.32$ \\
\hline $6 \sigma$ & & $0.09 /-0.06$ & $0.21 / 0.00$ & $0.41 / 0.04$ & $0.70 / 0.05$ \\
\hline $\begin{array}{l}\text { Multi-Peak } \\
\text { Assumption }\end{array}$ & $\begin{array}{c}\text { Relative } \\
\text { Abundance }\end{array}$ & $1 / 1$ & $0.5 / 1$ & $0.25 / 1$ & $0.125 / 1$ \\
\hline \multicolumn{6}{|l|}{$\begin{array}{c}\text { Peak } \\
\text { Separation }\end{array}$} \\
\hline $0 \sigma$ & & $80.11 /-79.47$ & $80.11 /-79.47$ & $80.11 /-79.47$ & $80.11 /-79.47$ \\
\hline $1 \sigma$ & & $39.88 /-39.24$ & $33.90 /-43.91$ & $27.68 /-46.94$ & $22.53 /-48.70$ \\
\hline $2 \sigma$ & & $16.95 /-16.38$ & $8.22 /-21.95$ & $-3.63 /-25.11$ & $-16.66 /-26.80$ \\
\hline $3 \sigma$ & & $6.06 /-5.66$ & $20.89 / 3.89$ & $48.25 / 11.63$ & $-29.35 /-12.69$ \\
\hline $4 \sigma$ & & $1.81 /-1.59$ & 4.87 / 0.27 & $9.29 / 1.25$ & $15.65 / 1.68$ \\
\hline $5 \sigma$ & & $0.44 /-0.36$ & $1.17 / 0.06$ & $2.13 / 0.22$ & $3.68 / 0.32$ \\
\hline $6 \sigma$ & & $0.09 /-0.06$ & $0.21 / 0.00$ & $0.41 / 0.04$ & $0.70 / 0.05$ \\
\hline
\end{tabular}

Table S3 Deviations of peak centroid calculations of simulated data in the assessment of the automated peak selection method used for calculation of mass defect peak areas (approximating peak boundaries by dropping a line to zero at local minima), means and full-width-half-max (FWHM). The simulated data consisted of two gaussian peaks with $\sigma=100$, with deviations for both peaks represented in each cell, separated by " / ". The single and multi-peak assumptions respectively reflect whether the simulated data was assumed to contain a single peak or multiple peaks. 


\begin{tabular}{|c|c|c|c|c|c|c|}
\hline \multicolumn{7}{|c|}{ Melittin-Incorporated DMPG Nanodisc Data } \\
\hline & $\begin{array}{c}\text { \# of } \\
\text { Melittin }\end{array}$ & $\begin{array}{c}\text { Mass Defect } \\
\text { (Expected) }\end{array}$ & $\begin{array}{c}\text { Mass Defect } \\
\text { (Peak } \\
\text { Integration) }\end{array}$ & $\begin{array}{l}\text { Standard } \\
\text { Deviation }\end{array}$ & FWHM & RMSD \\
\hline \multicolumn{7}{|l|}{ UniDec } \\
\hline 0 & 0 & 72.4253 & 48.1208 & 33.4451 & 78.7571 & 24.3045 \\
\hline \multirow[t]{3}{*}{1.5} & 0 & 72.4253 & 62.9280 & 39.0801 & 92.0266 & 35.8189 \\
\hline & 1 & 251.2879 & 289.6118 & 58.3700 & 137.4509 & \\
\hline & 2 & 430.1505 & 478.0050 & 39.9752 & 94.1343 & \\
\hline \multirow[t]{3}{*}{3} & 0 & 72.4253 & 60.4752 & 27.3609 & 64.4301 & 20.0798 \\
\hline & 1 & 251.2879 & 255.3657 & 33.6639 & 79.2724 & \\
\hline & 2 & 430.1505 & 462.5566 & 61.9354 & 145.8468 & \\
\hline \multirow[t]{4}{*}{6} & 0 & 72.4253 & 79.1632 & 40.4490 & 95.2501 & 11.5677 \\
\hline & 1 & 251.2879 & 234.0467 & 25.7910 & 60.7331 & \\
\hline & 2 & 430.1505 & 418.3673 & 26.3267 & 61.9945 & \\
\hline & 3 & 609.0131 & 601.6822 & 26.5076 & 62.4206 & \\
\hline \multirow[t]{4}{*}{9} & 4 & 120.9090 & 131.5617 & 28.7969 & 67.8115 & 21.9732 \\
\hline & 5 & 299.7716 & 312.1929 & 30.2316 & 71.1899 & \\
\hline & 6 & 478.6341 & 438.2619 & 34.9606 & 82.3258 & \\
\hline & 3 & 609.0131 & 614.8098 & 29.0172 & 68.3304 & \\
\hline \multirow[t]{4}{*}{12} & 4 & 120.9090 & 141.2823 & 36.6193 & 86.2319 & 16.8861 \\
\hline & 5 & 299.7716 & 312.1315 & 37.9574 & 89.3828 & \\
\hline & 6 & 478.6341 & 467.4528 & 39.8328 & 93.7991 & \\
\hline & 7 & 657.4967 & 636.3380 & 35.7912 & 84.2818 & \\
\hline \multirow[t]{4}{*}{24} & 4 & 120.9090 & 166.9398 & 38.3100 & 90.2131 & 23.9999 \\
\hline & 5 & 299.7716 & 305.9560 & 24.8769 & 58.5807 & \\
\hline & 6 & 478.6341 & 485.1766 & 24.0776 & 56.6984 & \\
\hline & 7 & 657.4967 & 0.6997 & 21.9070 & 51.5870 & \\
\hline \multicolumn{7}{|l|}{ iFAMS } \\
\hline 0 & 0 & 72.4253 & 64.9467 & 42.9032 & 101.0292 & 7.4786 \\
\hline \multirow[t]{3}{*}{1.5} & 0 & 72.4253 & 66.7901 & 48.1732 & 113.4392 & 19.8326 \\
\hline & 1 & 251.2879 & 281.1103 & 28.0318 & 66.0098 & \\
\hline & 2 & 430.1505 & 414.0611 & 22.2513 & 52.3978 & \\
\hline \multirow[t]{3}{*}{3} & 0 & 72.4253 & 78.6316 & 30.3582 & 71.4882 & 17.0410 \\
\hline & 1 & 251.2879 & 265.3121 & 29.5446 & 69.5723 & \\
\hline & 2 & 430.1505 & 455.3694 & 28.9354 & 68.1377 & \\
\hline \multirow[t]{4}{*}{6} & 0 & 72.4253 & 78.4087 & 26.1854 & 61.6618 & 3.5184 \\
\hline & 1 & 251.2879 & 247.6519 & 28.4922 & 67.0940 & \\
\hline & 2 & 430.1505 & 430.3194 & 30.9833 & 72.9601 & \\
\hline & 3 & 609.0131 & 609.6964 & 24.9687 & 58.7967 & \\
\hline \multirow[t]{4}{*}{9} & 4 & 120.9090 & 148.7955 & 29.0347 & 68.3716 & 21.5303 \\
\hline & 5 & 299.7716 & 317.0059 & 28.5935 & 67.3325 & \\
\hline & 6 & 478.6341 & 483.3089 & 25.1844 & 59.3048 & \\
\hline & 3 & 609.0131 & 636.5390 & 30.7236 & 72.3486 & \\
\hline \multirow[t]{4}{*}{12} & 4 & 120.9090 & 157.6944 & 29.5105 & 69.4920 & 22.4782 \\
\hline & 5 & 299.7716 & 322.9966 & 29.8685 & 70.3350 & \\
\hline & 6 & 478.6341 & 489.9335 & 31.3901 & 73.9181 & \\
\hline & 7 & 657.4967 & 656.5886 & 30.6896 & 72.2685 & \\
\hline \multirow[t]{3}{*}{24} & 4 & 120.9090 & 161.5878 & 28.4118 & 66.9046 & 27.5792 \\
\hline & 5 & 299.7716 & 323.0740 & 30.4655 & 71.7408 & \\
\hline & 6 & 478.6341 & 500.3669 & 29.5352 & 69.5501 & \\
\hline
\end{tabular}




\begin{tabular}{|c|c|c|c|c|c|}
\hline & 7 & 657.4967 & & 64.7646 & \\
\hline \multicolumn{6}{|c|}{ Mixed-MSP DMPC Nanodisc Data } \\
\hline & $\begin{array}{c}\text { Mass Defect } \\
\text { (Expected) }\end{array}$ & $\begin{array}{l}\text { Mass Defect (Peak } \\
\text { Integration) }\end{array}$ & $\begin{array}{l}\text { Standard } \\
\text { Deviation }\end{array}$ & FWHM & RMSD \\
\hline \multicolumn{6}{|l|}{ UniDec } \\
\hline \multirow{2}{*}{$\begin{array}{l}\text { Histidine- } \\
\text { tagged only }\end{array}$} & 514.4000 & 617.3985 & 121.7994 & 286.8157 & 102.9985 \\
\hline & & & & & \\
\hline \multirow{2}{*}{$\begin{array}{l}\text { His-tag cleaved } \\
\text { only }\end{array}$} & 24.3000 & 668.0286 & 94.19822 & 221.8199 & 34.17143 \\
\hline & & & & & \\
\hline \multirow{3}{*}{$\begin{array}{l}\text { Mixture } \\
\text { Ambient }\end{array}$} & 281.5000 & 272.2444 & 40.82698 & 96.1402 & 38.61799 \\
\hline & 514.4000 & 456.4018 & 40.52664 & 95.43293 & \\
\hline & 608.3000 & 670.1908 & 66.94955 & 157.6541 & \\
\hline \multirow{3}{*}{$\begin{array}{c}\text { Mixture } \\
90^{\circ} \mathrm{C} \text { for } \\
10 \text { minutes }\end{array}$} & 281.5000 & 278.2126 & 41.57944 & 97.91209 & 24.16901 \\
\hline & 608.3000 & 642.3217 & 96.37299 & 226.9411 & \\
\hline & & & & & \\
\hline \multirow{3}{*}{$\begin{array}{c}\text { Mixture } \\
80^{\circ} \mathrm{C} \text { for } \\
60 \text { minutes }\end{array}$} & 514.4000 & 445.7393 & 41.72021 & 98.24359 & 62.39332 \\
\hline & 608.3000 & 663.7217 & 83.5257 & 196.688 & \\
\hline & & & & & \\
\hline \multicolumn{6}{|l|}{ iFAMS } \\
\hline \multirow{2}{*}{$\begin{array}{l}\text { Histidine- } \\
\text { tagged only }\end{array}$} & 514.4000 & 611.1677 & 124.0128 & 292.0279 & 96.76774 \\
\hline & & & & & \\
\hline \multirow{2}{*}{$\begin{array}{l}\text { His-tag cleaved } \\
\text { only }\end{array}$} & 24.3000 & 13.20665 & 110.7438 & 260.7817 & 11.09335 \\
\hline & & & & & \\
\hline \multirow{3}{*}{$\begin{array}{l}\text { Mixture } \\
\text { Ambient }\end{array}$} & 281.5000 & 302.1674 & 41.05884 & 96.68617 & 16.36484 \\
\hline & 514.4000 & 496.8346 & 36.28081 & 85.43478 & \\
\hline & 24.3000 & 32.53034 & 62.16065 & 146.3771 & \\
\hline \multirow{3}{*}{$\begin{array}{c}\text { Mixture } \\
90^{\circ} \mathrm{C} \text { for } \\
10 \text { minutes }\end{array}$} & 281.5000 & 249.8713 & 4.766691 & 11.2247 & 35.5234 \\
\hline & 608.3000 & 647.3313 & 111.9027 & 263.5106 & \\
\hline & & & & & \\
\hline \multirow{3}{*}{$\begin{array}{l}\text { Mixture } \\
80^{\circ} \mathrm{C} \text { for } \\
60 \text { minutes }\end{array}$} & 514.4000 & 461.7888 & 44.15045 & 103.9664 & 59.98376 \\
\hline & 608.3000 & 674.8444 & 81.62567 & 192.2138 & \\
\hline & & & & & \\
\hline
\end{tabular}

Table S4 Statistical information for assessment of MMD analysis mass defect accuracy as expected from empirically determined masses. Peak integration for calculating peak centroids and standard deviations was performed with the assumption each peak begins and ends abruptly at local minima in the mass defect profile. Data is presented only for peaks selected by an automated peak selection method and which are shared between the two MMD analysis methods. 

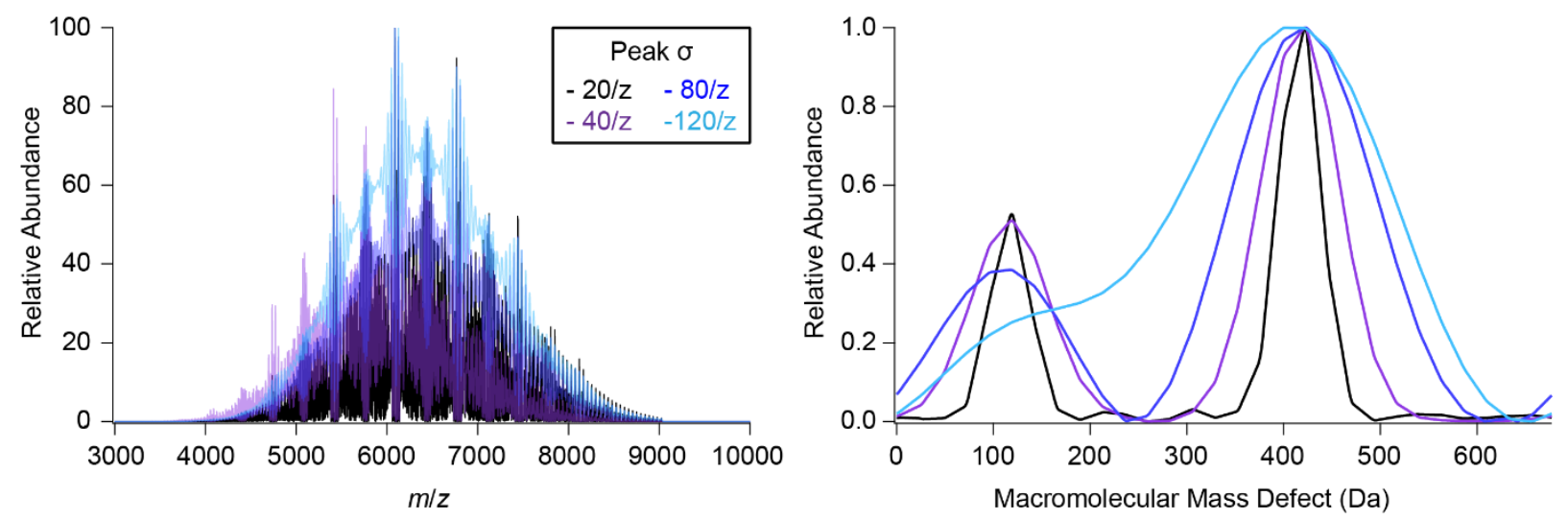

Figure S1 Comparison of simulated spectra and MMD profiles as peak width is varied. Wider peaks in the mass spectra lead to greater superposition, visually appearing as a higher curved baseline. The reduced resolution of the simulated mass spectra is mirrored in their MMD profiles, and inconsistent peak height ratios justifies our integral treatment of relative mass defect abundance (see Table S3) 


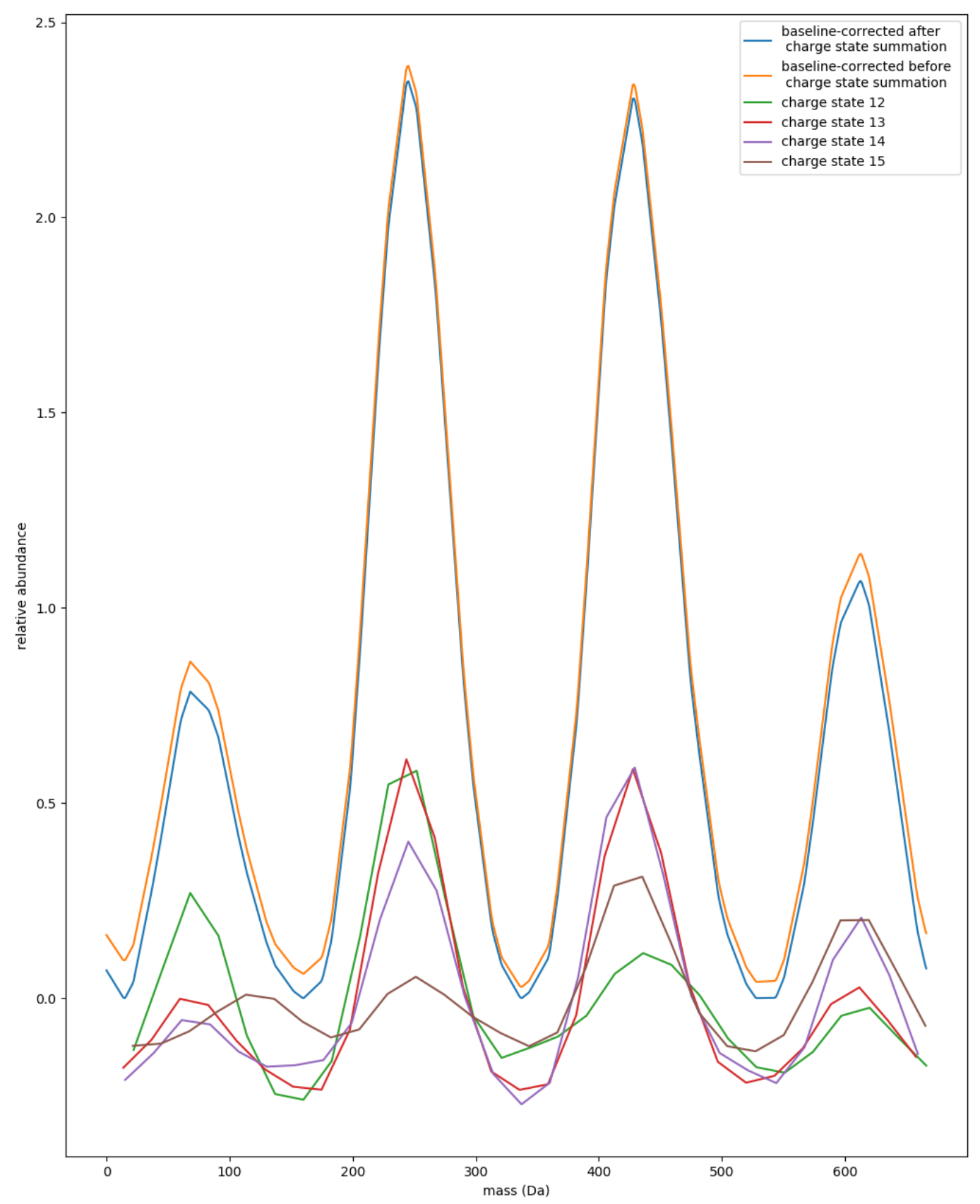

Figure S2 Charge-state-specific (green, red, purple and brown traces) and total MMD profiles (blue and orange traces) for melittin-embedded Nanodiscs prepared at a bulk melittin:Nanodisc ratio of 6:1. Baseline correction was applied to either the individual charge state profiles (orange) or their interpolated sum (blue) with negligible difference, so the MMD profile baseline correction implemented in iFAMS 6.1 is arbitrarily applied after summation of the charge-statespecific profiles. 


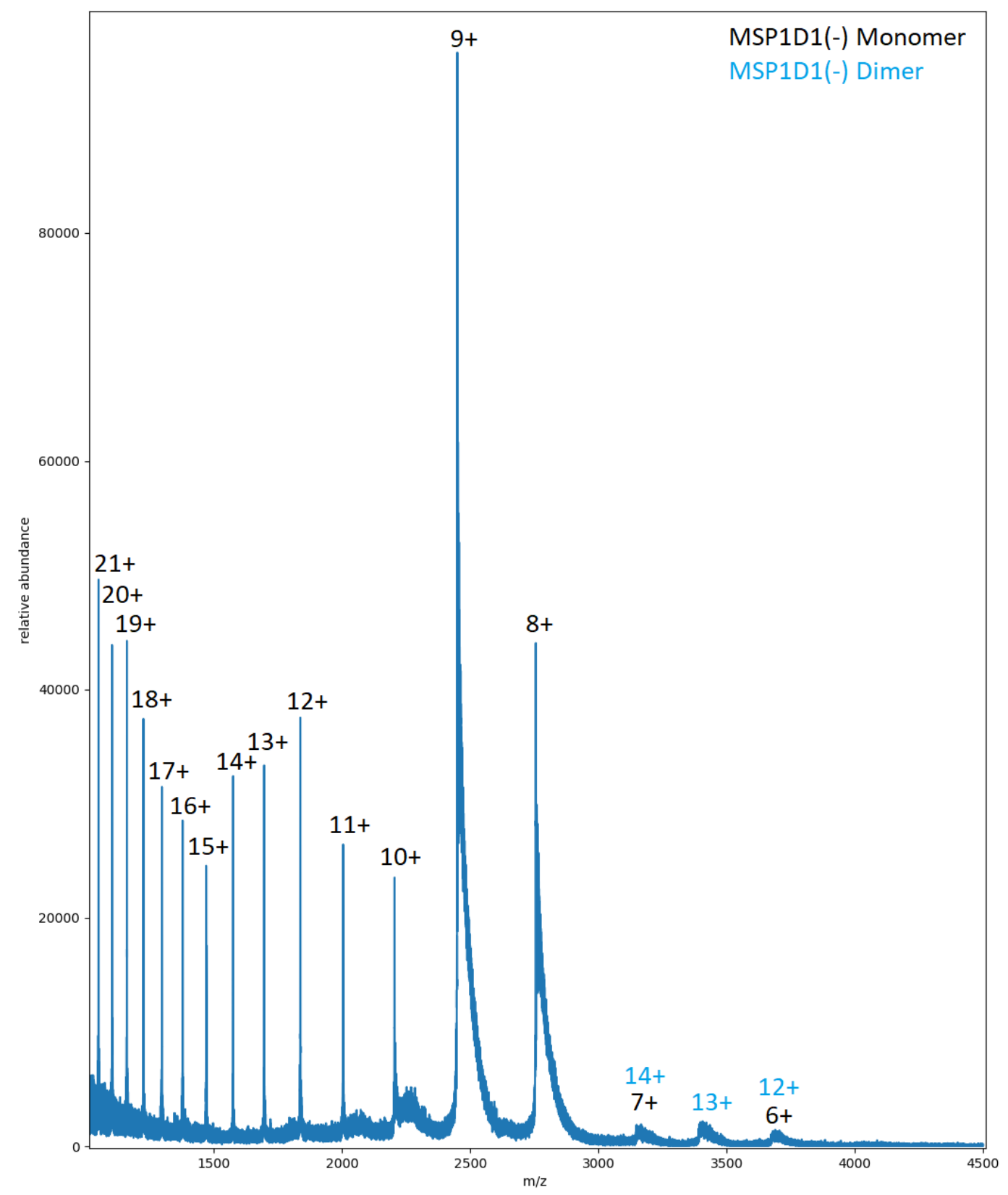

Figure S3 Example native mass spectrum of membrane scaffold protein MSP1D1(-), taken with a trap voltage of $5 \mathrm{~V}$ and trap gas flow rate of $3 \mathrm{~mL} / \mathrm{min}$, showing both native monomer (black charge states) and dimer (blue charge states) signals. The presence of higher charge states ( 11$21+$ ) indicates some partially unfolded monomer population. 


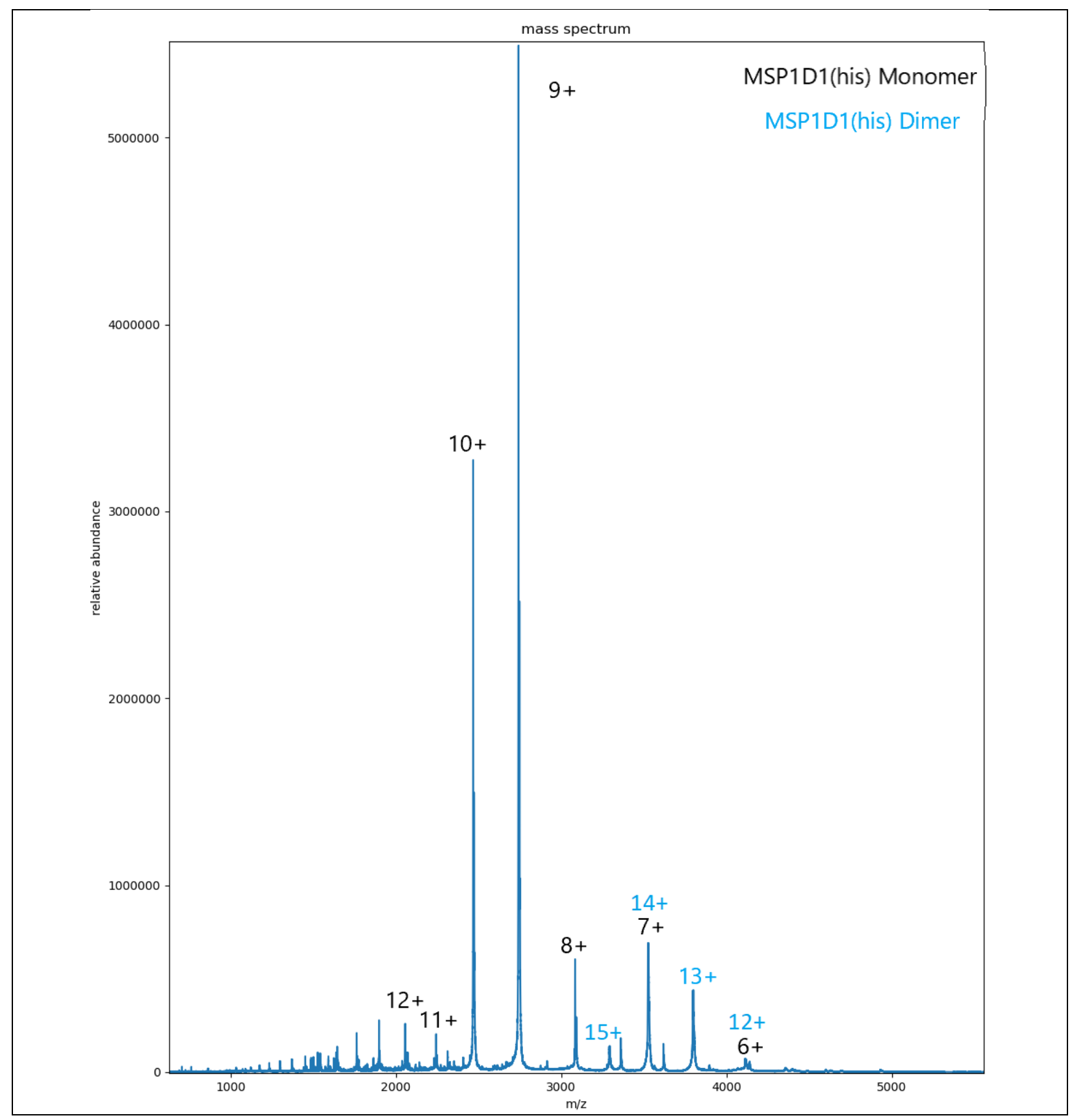

Figure S4 Example native mass spectrum of membrane scaffold protein MSP1D1His, taken with a trap voltage of $25 \mathrm{~V}$ and trap gas flow rate of $5 \mathrm{~mL} / \mathrm{min}$, showing both native monomer (black charge states) and dimer (blue charge states) signals. The presence of higher charge states $(\sim 11+$ and above) indicates some partially unfolded monomer population. 